\title{
Destruction of Perfluorinated Compounds (PFCs)
}

\section{PFCs Extent of Problem}

- Widely used in aerospace, automotive, building and construction, electronics, and consumer products

- Found in tap water of over $15 \mathrm{M}$ Americans; detectable levels in blood of $96 \%$ of those tested

- Linked to public health issues like cancer; EPA health advisory of 70 parts per trillion

\section{Problem with Conventional} Water Treatment Technologies

- Only concentrate PFCs, don't destroy

- Destruction of concentrates is expensive

- Don't work on short chain PFCs

\section{Solution to Problem}

- Electron beams can destroy PFCs

- Fermi National Accelerator Laboratory brings 50 years of groundbreaking accelerator expertise to bear to solve the PFC problem via a novel electron beam accelerator.

\section{Benefits of Novel Water Treatment Tool}

- Can completely destroy PFCs

- Can treat other known and emerging contaminants in water

- Can treat multiple contaminants simultaneously

- Technology mitigates future treatment, cost, compliance, and risk issues

- Avoids the long ramp up period to develop new solutions

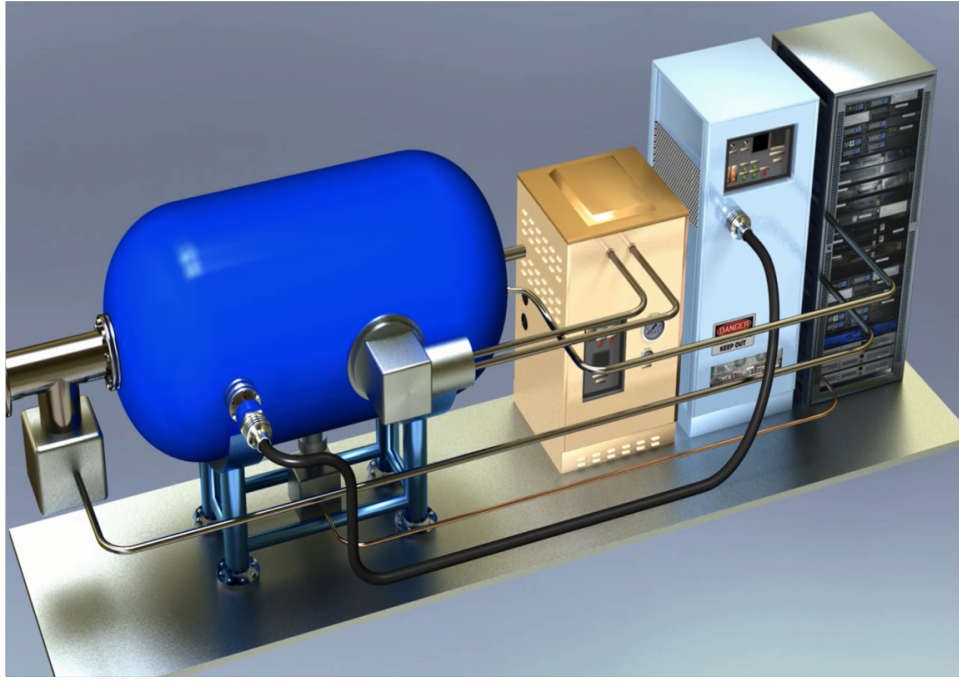

Department of Energy's Fermilab Universal Water Treatment Tool

\section{Advances to "E-beam" Technology}

- Can treat 200k gal/day (5X Improvement)

- Small, 5x7x13 feet (was 3 Story Building)

- $30 \%$ reduced operating cost

- $50 \%$ reduced power requirement

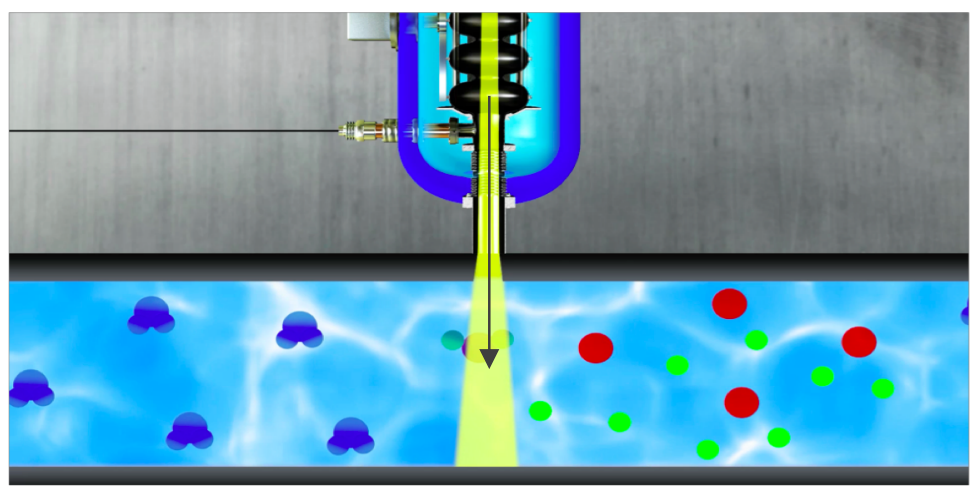

During the treatment process, electrons create charged species that are very effective at breaking down many complex contaminants. 